\title{
Family Assessment in the Medical Setting
}

\author{
Gabor I. Keitner \\ Rhode Island and Miriam Hospitals, Brown University, Providence, R.I., USA
}

\begin{abstract}
Acute and chronic illness exists in a social context. A biopsychosocial assessment should include an evaluation of the patient's social situation, the nature of the patient's interpersonal connections, and his/her family's functioning. Families can influence health by direct biological pathways, health behavior pathways, and psychophysiological pathways. There are a large number of family-based risks factors as well as many protective relational factors that influence the onset and course of illness. A family assessment is the first step in determining both the need for further intervention and the specific areas of family life that might need to be addressed. A family assessment provides information about the social substrate for the evolution of the presenting complaint as well as information to better understand the patient's problems. There are many ways to assess families, and there are a variety of family assessment instruments to help with the assessments. Clinician will be best served by becoming familiar and comfortable with at least one assessment model that is consistent, structured, and evaluates a wide range of family functions. Such an assessment can be incorporated into routine clinical care. A good family assessment can be therapeutic in and of itself even if the decision is made that no further family intervention is indicated. Copyright $\odot 2012$ S. Karger AG, Basel
\end{abstract}

Acute and chronic illness exists in a social context. The importance of developing a biopsychosocial formulation leading to treatment that addresses the biological, psychological and social components of a patient's illness is well established. A biopsychosocial assessment should include an evaluation of the patient's social situation, the nature of the patient's interpersonal connections and particularly his/her family's functioning. A patient's most important resource is the family, the group that helps the patient to function [1]. Families have a powerful influence on health equal to traditional medical risk factors [2].

Improved health care has led to prolonged periods of living with disease. Longer periods of illness increase strain on patients and their significant others leading to caregiver burden which in turn impacts on the ability of caregivers to provide support for ill family members [3]. Emotional support (being listened to, cared for and empathized with) is the most important and influential type of support provided by 
Table 1. Family relational factors that have adverse effects on illness

- High interpersonal conflict

- Low relationship satisfaction

- Poor problem solving skills

- High levels of criticism and blame

- Intrafamilial hostility

- Poor family organization

- Inconsistent family structure

- Family perfectionism and rigidity

- Low family cohesion and closeness

- Lack of extrafamilial support system

families. Negative, critical or hostile family relationships, in turn, have a stronger, detrimental, influence on health than positive or supportive relationships [2].

Families can influence health by direct biological pathways, health behavior pathways and psychophysiological pathways. Examples of direct biological pathways include spreading of infectious agents, sharing similar toxic environments and genetic vulnerabilities. Health behavior pathways include lifestyle behaviors such as smoking, exercise, diet and substance abuse. Healthcare behaviors include adherence to treatment as well as family caregiving. Pathophysiological pathways refer to the effects of family environment on neuroendocrine and psychoimmunological pathways [2].

There are a large number of family-based risk factors that adversely influence the onset and course of illness. These include: poor conflict resolution, low relationship satisfaction, high interpersonal conflict, criticism and blame, intra-familial hostility, lack of congruence in disease beliefs and expectations, poor problem solving, extrafamilial stress, lack of extra-familial support systems, poor organization, inconsistent family structure, family perfectionism and rigidity, low cohesion and closeness, and presence of psychopathology in family members [4] (table 1). Conversely, there are many protective relational factors. These include: good communications, good problem solving skills, adaptability, clear roles, achievement of family developmental tasks, mutual support, open expression of appreciation, commitment to the family, extra familial social connections, spending time together, religious and spiritual orientation [5] (table 2).

Most patients prefer that physicians involve their family in their medical care. Family members can provide valuable information about the patient's functioning at home and can help patients to comply with treatment recommendations [1]. Families can also help to keep track of medication side effects and prodromal and residual symptoms. They can help in sharing responsibilities, lessen the patient's anxieties and facilitate as well as encourage communication between providers [6]. Family interventions have been found to be helpful in the management of chronic physical 
- Good communication

- Adaptability

- Clear rules

- Mutual support

- Open expression of appreciation

- Commitment to the family

- Spending time together

- Good problem solving skills

- Extrafamilial social connections

diseases. Hartmann et al. [7] undertook a meta-analysis of 52 methodologically sound randomized clinical trials involving 8,896 patients with cardiovascular diseases, cancer, arthritis, diabetes, AIDS and systemic lupus erythematosus. They found that the physical health of the patient was significantly improved with family interventions compared to standard treatment. They also noted that the mental health of the medically ill patient was significantly improved with family interventions in comparison to standard treatment. Family interventions, in addition, had a significant effect on improving the health of other family members.

A comprehensive biopsychosocial approach to patient care involves a shift of focus from the patient to the social setting in which the disease takes place. A familyfocused approach to the management of illness, particularly chronic illness, emphasizes assessing the relational context, and includes the family as potential targets for intervention. A family-focused approach addresses the educational, relational and personal needs of the patient and their family members, and includes the patient and their family members in treatment and the assessment of outcome [5]. The first step in this process is undertaking a family assessment.

Family assessment and intervention in medical settings for somatic and/or psychosomatic illnesses is the exception rather than the rule in spite of the evidence for its importance for the treatment and management of diseases. The medical home has been identified as the main vehicle for providing 'comprehensive primary care in a way that facilitates partnerships between individual patients and their personal providers and when appropriate the patient's family' [8]. There is a general recognition of the important role that families can play in supporting or undermining treatment efforts, but even the concept of the medical home relegates the family to a peripheral position. The major goals of the medical home are to ensure a personal physician, a physician-directed medical practice, a whole person orientation, coordinated/integrated care, emphasis on quality and safety and enhanced access to care. An additional goal should be the involvement of the patient's family in the assessment and treatment of their illness. 


\section{Family Assessment}

The essential task in meeting with family members for the first time in family assessment is to evaluate and assess their functioning in the context of understanding their problem. Family assessment is the first step in determining both the need for further interventions and the specific areas of family life that might need to be addressed. The family assessment provides not only information about the social system, the substrate for the evolution of the problem, and how it may be currently contributing to improving or maintaining the problem, but also information to better understand the patient's problem(s). Family assessment should focus on adjustments related to the diagnosis of medical illness, clarification of treatment options, and collaboration in carrying out the treatment plan. A proper assessment should also identify family strengths.

\section{Individual Life Cycle Tasks}

A family faces different demands at different points of time. Understanding the stage of the family life cycle helps in understanding both the challenges that a family faces and in evaluating their effectiveness in meeting those challenges. Adult developmental phases can be divided roughly into early adulthood (age 20-40 years), mid adulthood (age 40-60 years), and older adulthood (age 60 years and older).

\section{Early Adulthood}

The tasks of early adulthood include launching from one's family of origin and developing a sense of identity, life structure, career track, and movement toward an intimate and committed relationship. Relationships with parents are renegotiated. The birth of children creates the need to be able to handle multiple responsibilities. The struggle is to find a balance between the pleasures and pressures of taking care of the family, advancing in the workplace, while finding enough time for individual pursuits.

\section{Mid-Adulthood}

In this stage, individuals become more comfortable with their multiple roles and, if successful, have a greater sense of effectiveness and competence. There may be a need to reevaluate work goals and relationships, leading at times to some mid-life transitions and anxiety surrounding this reevaluation. There is a tendency over time to accept what one has become and to begin to deal with the reality of aging and mortality. It is also a time when individuals may need to take care not only of their children but also their parents. 


\section{Older Adulthood}

In this stage, individuals begin to review their lives more systematically. They may gradually transition from feeling a need to be in charge to providing more support for the next generation. Medical problems and the reality of physical limitations as a result of aging need new accommodations. With the possibility of retirement, finding new meaning in life becomes important.

\section{Family Life Cycle Tasks}

Marriages and families also go through various stages of evolution and development, each of which presents its own unique challenges and satisfactions. It is helpful to have some conception of these different phases to better understand the issues that a particular family may be presenting.

\section{Courtship and Early Marriage}

Couple formation is best done when both individuals have completed the task of restructuring relationships with their parents, learned enough about themselves to become aware of their characteristic problems and had enough freedom and adventure that the demands of an intense relationship feel comforting rather than constrictive. They must establish an identity as a couple, developed effective ways of communicating and solving problems and begin to establish a mutual pattern of relating to parents, friends and work. Common stress points in early adult marriages are childbearing, attempting an egalitarian marriage in a nonegalitarian culture, and enduring midlife transitions as one or both partners go through a period of reevaluating themselves. Individuals often enter into a marriage with unrealistic expectations. Individuals tend to want somebody who is familiar and comfortable - in other words, like themselves - while at the same time hoping that the other person will complement and complete those parts of themselves that they feel are deficient or problematic. A lack of acceptance of individual differences between couples leads to attempts by one individual to make the other more like them, often resulting in conflict and disappointment.

\section{Children}

With the arrival of children, families tend to become closer, with an intense inward focus on the children. Roles need to be redefined. Family boundaries need to be loosened to allow extended family members to participate in the enjoyment of and the 
sharing of responsibilities of caring for the children. It can be difficult for parents to find sufficient time for each other with multiple demands of work, home maintenance and parenting. As children become adolescents, they press for greater autonomy. Families at this stage must be strong, flexible and able to support growth. They need to be able to maintain boundaries and limits, while at the same time allowing their children increasing exploration and autonomy.

\section{Empty Nest}

As children leave home, parents need to be able to redefine their roles and priorities. It is an opportunity for parents to spend more time together, to feel less pressure, and to explore individual interests. It is also a time of potential distress when interpersonal problems between the parents that had been sidelined by focusing on the needs of the children may surface. There is a need at this stage to redefine goals and meaning, while at the same time finding ways of connecting more closely to one's partner.

\section{Retirement}

Retirement requires individuals to redefine their identity and place in society. The solution to these challenges inevitably affects relationships. Finding new interests and goals and feeling connected and useful allow for successful enjoyment of new leisure time. Couples have to find ways of managing the increased amount of time they spend together. They have to find ways of being together that are supportive without being enmeshed or too confining. The health and financial stability of the couple will have a major impact on their ability to experience this stage of their lives in a satisfying way.

\section{Death and Grieving}

As couples age and health problems increase, they will increasingly have to deal with the death of friends, their partner and eventually themselves. Individuals have to come to terms with their own mortality and find ways of resolving issues of importance to them before dying. They are likely to be worried about the health of their partner and experience fears of loss, dependence and isolation. Couples need to find ways of discussing their concerns and plan together how they want to deal with the problems they are likely to face. The involvement of children and extended family members can ease many concerns. Unresolved family problems can make this process difficult. 


\section{Including the Family in the Evaluation of a Patient}

When should family assessments take place in the context of providing routine clinical care? Is a family assessment a routine component of the evaluation of a patient's presenting problem(s) or should it be scheduled as a separate assessment process? Does it matter if the presenting problem is primarily physical, emotional or psychosomatic? The answer to these questions is going to be determined largely by the orientation, interest and skill level of the assessing clinician as well as the reality of time constraints in a busy clinical practice.

In an ideal situation, the patient's significant other(s) should be seen with the patient if a patient presents for an initial evaluation of a complicated medical problem or if the patient is scheduled for follow-up visits for persisting or chronic problems. During that visit, a brief assessment of the nature of the patient's relationships, following the guidelines outlined bellow, will disclose whether a more comprehensive family assessment is indicated. At a minimum, such a preliminary meeting may highlight new and helpful information to guide treatment and co-opt the significant other in supporting the treatment plan or make evident the problems that the patient is likely to encounter at home. If the initial meeting with the patient's significant other suggests more significant relationship issues then a comprehensive family assessment session can be scheduled. The initial visit should not take much longer than a usual first assessment or a usual follow-up session. A comprehensive family assessment session can take 30-90 min depending on the skill of the clinician and the complexity of the family's problems.

\section{Assessment of the Family}

There are three main goals when conducting a family assessment. The first is to orient the family to the interview process and to establish an open and collaborative relationship with the family. The second goal is to have family members identify all current problems in the family including the problem(s) that precipitated the meeting. The third goal is to identify the families' transactional style that appears to be related to the family's functioning. Dysfunctional transactional patterns are repetitive interactional processes that prevent effective resolution of ongoing interpersonal problems. The amount of time taken to assess the family, as well as the number of assessment sessions, will vary according to the level of experience of the evaluator and the nature of the family's problem(s). It can range from $30 \mathrm{~min}$ to $2 \mathrm{~h}$.

There are many different ways to assess a family and many different kinds of information that can be gathered. Some clinicians begin with a long history of the family's life, connections and evolution as a unit. They gather information on families of origin and may develop a genogram. Other clinicians are more interested in a here and now view of how the family functions and the current problems they are dealing with. Still, 
Table 3. General principles

- Include as many family members as possible

- Establish connection with all family members

- Do not blame the family

- Do not identify with the patient's perspective

- Evaluate a wide range of family functions

- Be sensitive to cultural and religious issues

other clinicians focus primarily on process issues in the family session. They address what they observe as the current family interactional pattern and assume that this is representative of the way in which the family deals with issues outside of the assessment session. All three approaches have merit, and they are not mutually exclusive. The challenge for the clinician is to integrate these three approaches in such a way as to meet the goals of the evaluation without getting unduly sidetracked by peripheral issues or take so long to complete the evaluation that the family loses interest.

The most important step in meeting with the family for the first time is to establish connection with the family (table 3). The family needs to feel understood, respected, and validated. They do not want to be blamed for their loved one's problems or judged for their perceived deficiencies. It is the job of the clinician to put families at ease and to make them feel comfortable enough to participate openly in the assessment process. The assessment is used to gain a better understanding of how everybody sees the problem(s) at hand, to gather information to allow for a more comprehensive formulation, to provide an opportunity for all members involved in trying to cope with the illness to ask questions and to solicit the help of all involved in setting up a meaningful treatment plan. As a rule, it is useful to have as many family members and significant others in the patient's social field included in an assessment as possible. In addition to the most immediate family members living with the patient, it is often helpful to meet with others who are significantly involved in the patient's life. These could involve extended family members, good friends, coworkers, mental healthcare providers, and spiritual counselors.

The assessment process consists of four identifiable components: Orientation, Data gathering, Problem description, and Problem clarification (table 4).

\section{Orientation}

The orientation explains the purpose of the evaluation and establishes goals for the assessment process. It is often helpful to start off the meeting with introductions of the participants. The meeting is normalized with an explanation that family meetings are a regular part of the assessment and treatment of most patients. This assures the 
- Orient the family to the assessment process

- Help all family members to identify all current problems

- Identify family transactional patterns

family that they are not being singled out for blame or that there is anything uniquely problematic about their family. The family can be further oriented by clarifying why they came for help, what they expected would happen, and what they would like to get out of the meeting. The therapist should provide feedback to the family about their responses to questions to make sure the family knows that they had been heard and understood correctly.

The clinician explains that the goal of the meeting is to provide an opportunity for all family members to identify what they see as problems and to bring up any areas of concern. The family's cooperation in the assessment process is enhanced by letting them know that they will also have an opportunity to ask questions of the therapist and to have input into the development of a treatment plan.

The clinician should obtain information about family members' names, ages, relationships within the family, and living arrangements. It is also helpful to ascertain the correct phase of the family life cycle to anticipate the kinds of problems and issues that the family may be struggling with in addition to the presenting problems.

\section{Data Gathering}

The clinician begins by asking family members what they think are the problems in the family. Each family member is given the opportunity to express his or her concerns without being interrupted by others. The clinician should spend enough time with each family member to ensure that each individual has an opportunity to outline any problems that he/she is concerned about. The challenge for the clinician at this stage is to not get sidetracked by beginning to deal with problems before everybody has had an opportunity to present their perspectives. The clinician also has to make sure the problems are not described in such great detail as to leave no time for the exploration of other problems and the concerns of other family members. Individual family members can be kept on track and their comments focused by feeding back to the individual what the clinician heard, to ensure that the correct information has been transmitted and to let the person know that his/her concerns have been understood. As a result, he or she does not have to give too may examples of the same problem. The clinician also reassures other family members that their concerns will also be addressed in due time as the goal is to develop a problem list from all family members. 
Table 5. Dimensions of family functioning

- Problem solving

- Communication

- Affective involvement

- Affective responsiveness

- Roles

- Behavior control

Listening carefully, limiting the amount of detail provided to exemplify each problem, summarizing the clinician's understanding of the problems to reassure family members that they have been heard, and giving everyone the opportunity to speak without interruption should allow for the development of an overview of the range of family concerns within a reasonable period of time. Once the presenting problems are clearly delineated by the various family members and once the clinician has summarized his or her understanding of these problems with consensus of all participants that the problems are understood and agreed upon, the therapist moves on to assess broader dimensions of family functioning [9].

\section{Overall Family Functioning}

The clinician orients the family to this new stage of the assessment process by letting them know that he/she will be asking a series of questions about different aspects of their family's life. These include: problem-solving, communication, affective involvement, affective responsiveness, roles, and behavior control [9] (table 5).

\section{Problem-Solving}

Problem-solving refers to a family's ability to resolve problems to a level that maintains effective family functioning. A family problem is one that threatens the integrity and functional capacity of the family and the family has difficulty solving. Family problems can be instrumental or affective. Instrumental problems refer to problems of everyday life, such as managing money and obtaining food, clothing and housing. Affective problems concern issues of emotion or feeling, such as anger or depression.

Questions about problem-solving skills of the family may be general, or, if the family has trouble abstracting about their problem-solving procedures, family members can be asked to think of family problems that have come up within the past few weeks to use as an example. Stages of effective problem-solving include identifying a problem, communicating about it with an appropriate person, developing alternatives, deciding on an alternative, acting on that decision and evaluating the process at its conclusion. 
The following questions may be helpful in exploring the family's effectiveness in managing problems: Who first notices problems? What was done after the problem was noticed? Did you discuss it with anybody? What did you decide to do about the problem? Did you think of any alternatives? Did you review how you dealt with the problem once you had taken care of it? How do you handle practical problems? How do you handle problems that involve emotions?

\section{Communication}

Communication refers to the verbal exchange of information within the family. Nonverbal communication is very important as well, but it is more difficult to quantify and monitor. Communication can be affective or instrumental. Families can have marked difficulties with affective communication while functioning very well in the instrumental area. Families who experience poor instrumental communication can rarely communicate effectively about emotional issues. Communication can also be thought of as clear versus masked. Masked communication tends to be muddied or vague. Communication can further be direct or indirect. Indirect communication tends to get deflected from the intended target to someone else. Families with poor functioning tend to have unclear and/or indirect form of communication.

The following are questions that can be used to assess communication in a family: Do you talk with one another? Can you talk about practical things with each other? Can you talk about emotional issues with each other? Do you feel that you can say what you want or do you have to be guarded about what you say? Can you tell things to each other directly or do you have to use someone else to let others know how you feel and think?

\section{Affective Involvement}

Affective involvement refers to the extent to which the family shows interest in and values the activities of individual family members. Families can lack involvement with each other and not show any interest in each other. They coexist in the same space without much connection. Some family members are narcissistically involved. The investment in others is only in terms of what that individual can get out of it without concern for the other. With empathic involvement, family members demonstrate true concern for the interest of others in the family, even though these concerns may be peripheral to their own interests. Over involvement and symbiotic involvement is overintrusive and overprotective, sometimes to the point that boundaries between family members are significantly blurred.

The following questions can help explore aspects of affective involvement: Who cares about what is important to you? Do you think that other family members are interested in you? Do they ever show too much interest? Do you think that others are truly interested in you because it is important to you or only because they think that they should be? Do you feel that other members of the family go their own way and do not care or notice what happens to you? 
- Provision of resources - food, shelter, clothing

- Nurture and support - warmth and reassurance

- Adult sexual gratification - mutually respectful

- Personal development - school, career, social skills

- Maintenance and management of the family system - decision making, dealing with other systems, financial planning

- Health-related functions - indentifying problems, appointments, compliance, promoting health behaviors

- Role allocation - appropriateness, fairness

- Role accountability - monitoring and corrective measures

\section{Affective Responsiveness}

Affective responsiveness refers to whether family members are able to respond to the full spectrum of feelings experienced in emotional life and whether the emotion experienced is consistent or appropriate with the stimulus, situation, or context. This dimension assesses an individual's capacity to a greater extent than do the other family dimensions. It refers to the person's capacity to experience particular kinds of emotions. It is assessed in order to determine whether family members tend to be overcome with feelings or are not sufficiently capable of experiencing them. It is useful to know if some of the family difficulties may be a function of particular individuals' under- or overresponsiveness to affective stimuli. Two types of affect are assessed. Welfare emotions consist of affection, warmth, tenderness, support, love, happiness and joy. Emergency emotions include fear, anger, sadness, disappointment and depression.

The following questions can elicit information regarding emotional responsiveness: Are you a family that responds to situations with a lot of feeling? Do you feel that you are a family that does not respond with enough emotions? Which kinds of emotions do you think that you overrespond or underrespond to? Do others sense that you do not experience feelings that you should feel? Are there any feelings that you experience more intensely than you think is reasonable given the situation?

\section{Roles}

Roles are the repetitive patterns of behavior by which family members fulfill family functions. Family functions include the provision of resources, nurturance and support, sexual gratification, personal development, and maintenance and management of the family system. These include decision making, boundaries and membership functions as well as household finances and management (table 6).

Questions to explore roles in the family include the following: How do you divide responsibilities? Who works and for how many hours? Who handles the money? Who buys the groceries and prepares the meals? Who does the housework? Who looks 
after the home and cars? Who oversees what happens with the children's education? Who gets involved with the schools? Who is involved in major decisions? Who has the final say? How do you decide who does what job? Do you feel that some people have too many jobs? Do any of you feel overburdened by your jobs? Are the responsibilities fairly shared between family members? If not, how would you like to see it done differently?

\section{Behavior Control}

The behavior control dimension evaluates the ways in which a family establishes rules about acceptable behavior related to physically dangerous situations, situations involving meeting and expressing psychological needs and drives, and situations involving socializing behavior between family members and people outside the family. It concerns parental discipline toward children as well as standards and expectations of behavior that adults set for each other. There are a variety of styles of behavior control including rigid behavior control, flexible behavior control, laissez-faire behavior control (where there are no standards or direction), and chaotic behavior control (where standards shift in a random and unpredictable fashion between rigid, flexible and laissez-fair and family members do not know which standards apply at any one time).

The following questions can be used to explore the behavior control dimension of family functioning: Do you have rules in your family about how to handle different situations? How do you handle dangerous situations? Do you have rules for table manners and for bedtime? Do you allow hitting or yelling at each other? Do you know what is expected of you in terms of behavior with people outside the family? Do you have rules about drinking? Driving too fast? Letting people know where you are when away from home? Are the rules clear? Are the rules the same for everybody? Can you discuss the rules to change them? Do you always know what the family expects? Do you know what to expect if the rules are broken?

It is important not to get side tracked by a particular problem before understanding the whole system. Often, further exploration of the family system allows the identification of dysfunctional patterns in which a similar problem surfaces in other areas of family functioning.

\section{Additional Investigations}

A comprehensive family assessment should not preclude a diagnostic workup that also includes psychological and biological information and data. For adults, this includes a psychosocial history, psychiatric examination, medical history and physical examination. Laboratory and radiology tests as well as neurological and psychological assessments should be ordered as indicated. Information from any of these sources may add to the understanding of the family's functioning. 


\section{Problem Description}

After the thorough assessment described above, family problems are likely to be clear to the clinician and to the family. It is helpful to group a variety of different problems into related clusters in order to focus the family and to avoid the risk of getting bogged down into excessive detail. What may seem to be many different problems often emanate from a few core problems.

\section{Problem Clarification}

The final step in the assessment process is to obtain agreement between the clinician and family members on the problems identified. If the clinician has been careful to listen to each family member and has checked out his or her understanding of the problems with the family, there should be good agreement on the final problem list. If there are disagreements about the problem list between the clinician and various family members these should be resolved before proceeding to any recommendations for treatment.

\section{Tools for Family Assessment}

A variety of instruments are available to evaluate families systematically. Family assessment instruments allow for numerical quantification of family functioning, so as to track change over time, make comparisons with other families and carry out quantitative research. There are subjective and objective family assessment tools.

\section{Subjective Family Rating Scales}

These scales are self-report paper and pencil or computer touch screen instruments filled out by individual family members. They elicit individual family members' views of their own family's functioning. Self-report instruments are cost-effective as family members can fill them out at their leisure, and the assessment does not require trained interviewer time. Most questionnaires can be filled out in less than $30 \mathrm{~min}$. They do not require manuals to complete, can be easily transported to a variety of settings and can be compared over time. One potential problem in understanding subjective perspectives of family functioning is how to integrate the views of different family members, especially if they are different from each other. One way is to average individual scores and arrive at an overall family score. Another way is to look at differences in the perceptions of various family members. The disadvantage of self-report instruments is that they are restricted to an internal perspective of family functioning 
Table 7. Self-report instruments of family functioning

- Dyadic Adjustment Scale

- Self-Report Family Inventory

- Family Environment Scale

- Family Adaptability and Cohesion Evaluation Scale III

- Family Assessment Device

and may not reflect the way a family appears to function to an outside observer. In addition, not all family assessment instruments are reliable or have been validated. Different scales also emphasize different aspects of family functioning or measure the same concept differently depending on the model from which they are derived.

There are a number of useful self-report measures of marital adjustment/satisfaction and family functioning. Family members can fill these out in the waiting room or at home, before the first session or during a course of treatment as a way of monitoring progress. The following are examples of commonly used instruments (table 7).

Dyadic Adjustment Scale [10]: Measures satisfaction, cohesion, consensus, and affectional expression in couples.

Self-Report Family Inventory [11]: Assesses conflict resolution, styles of relating, intergenerational boundaries, and family competence.

Family Environment Scale [12]: Assesses relationship (cohesion, expressiveness, conflict), personal growth (independence, achievement, mortality/religion), and system maintenance (organization, control).

Family Adaptability and Cohesion Evaluation Scale III [13]: Measures adaptability (rules, power structure, roles) and cohesion (emotional bonding, autonomy, boundaries).

Family Assessment Device [14]: Assesses the dimensions of the McMaster Model of Family Functioning - problem solving, communication, role allocation, affective responsiveness, affective involvement, behavior control, and general functioning.

The Dyadic Adjustment Scale can be filled out in 5-10 min and provides a measure of relationship satisfaction/dissatisfaction. The Family Assessment Device can also be filled out fairly quickly (15-20 min) and explores a wider range of family functions. It has good validity and reliability, has been used in numerous outcome studies and has been translated into more than 25 languages.

\section{Externally Rated Instruments of Family Functioning}

These instruments are administered by trained interviewers and provide a more objective view of the family's functioning. These structured or semistructured interview instruments are independent of the family's tendency to want to see themselves 
Table 8. Externally rated instruments of family functioning

- GARF

- Beaver's International Styles Scale

- Camberwell Family Interview

- McMaster Clinical Rating Scale

in a particular way. They provide more reliable assessments for comparisons between different families and comparisons with established population norms. A disadvantage of externally rated family assessment instruments is their relative expense. Interviewers have to be trained to rate families reliably and these instruments take a longer time to administer.

Observer-rated couples and family interaction instruments provide a more objective perspective of a family's way of dealing with each other and the world around them. The following are some of the commonly used instruments (table 8).

\section{Global Assessment of Relational Functioning}

The Global Assessment of Relational Functioning (GARF) scale is similar to the individual focused Global Assessment of Functioning but focuses instead on relational adjustment and also on the quality of the family environment [15]. The GARF measures relational functioning on a scale of $1-99$. Scores of $1-28$ apply to a family that is too dysfunctional to retain continuity, contact, and attachment to each other; scores of 21-40 described a family that is obviously and seriously dysfunctional; scores of 41-60 define a family that has occasional times of satisfactory functioning but unsatisfactory relationships predominate; scores of 61-80 suggest that family functioning is somewhat unsatisfactory; scores of 81-99 define a family where family functioning is satisfactory. Three areas are assessed: interactional problem solving, organization and emotional climate. The GARF allows a clinician to document relational functioning on a scale that includes healthy family functioning and is an important step toward the recognition of family strengths as important components of the patient's presentation.

\section{Beaver's Interactional Styles Scale}

The Beaver's Interactional Styles Scale [11] evaluates a family's competence and style. It also assesses power, parental coalitions, clarity of expression, conflict, negotiation, responsibility, and empathy. 
The Camberwell Family Interview [16] measures expressed emotion, the amount of criticism expressed by family members about each other, and emotional over involvement (the extent to which family members are involved in each other's lives and concerns).

\section{McMaster Clinical Rating Scale}

The McMaster Clinical Rating Scale [17] is an interviewer assessment (with the aid of the McMaster Structured Interview of Family Functioning, if desired) of a family's communications, problem solving, affective involvement, affective responsiveness, roles, behavior control, and general functioning.

The GARF is easy to learn and can help to quantify overall family functioning. The McMaster Clinical Scale takes more time to learn and administer, but provides a more detailed assessment of a wide range of family functions. It is interconnected with the McMaster Model of Family Functioning, the Family Assessment Device and the Problem Centered Systems Therapy of the Family allowing for a comprehensive integration of a model of family functioning, ways of assessing it and a treatment approach for dealing with identified problems.

There is no absolute advantage to either an external or internal perspective on family functioning. Both perspectives are important, and each may be relatively more useful to answer different kinds of questions. A family's view of itself for instance may be just as important as an external observer's evaluation of the family even if the two views are different. A discrepancy between an outside evaluator and family members' perception of their family's functioning and differences between individual family members' view of their own family's functioning may provide particularly useful clinical information about that family's problems over and above the specific results of either form of assessment. The information from both kinds of assessments can be complementary to each other. Which one is used will depend on the questions being asked and the time and resources available to undertake the evaluation.

\section{General Assessment Issues}

Throughout the assessment process, the therapist should also identify potentially dysfunctional transactional patterns. These patterns are repetitive interactional processes that prevent effective resolution of ongoing interpersonal problems. If the pattern recurs, it is often in association with the presenting problem. A transactional pattern that is disruptive may need to be identified early in order to allow for the assessment to continue without being derailed. The clinician however should not be 
sidetracked with trying to deal with these patterns too early in the assessment process before a full understanding of the family has been achieved.

In the case of a crisis (suicidality, homicidality, spousal abuse, acute substance abuse), a comprehensive assessment may need to be suspended until the acute crisis has been contained and managed. It is also important to learn the family's functioning on a day-to-day basis and not just when reacting to a crisis situation.

Some family members may be overly talkative and controlling. It is important for the clinician not to become engaged in a power struggle with family members about the direction of the assessment. The clinician needs to respectfully focus the assessment to ensure that all family members have an opportunity to express their own perspectives. Different individuals in the family may have very different ideas of what is happening in the family, but all perspectives are legitimate.

Some families, in contrast, are very quiet and reluctant to participate in a family discussion. The clinician has to be careful in this situation to not fill in the vacuum by taking on too much responsibility for defining the family's problems. The clinician should identify that one of the problems in the family is a lack of ability or willingness to contribute to the understanding and exploration of their problems. If the family is still unwilling to participate, the clinician has to outline the reality that without their participation and help it will not be possible to arrive at a clear understanding of the issues at hand and a family assessment cannot continue. The clinician may have to be more directive if the family members are unable to engage more fully in the assessment process because of limitations in social competence and/or intellectual capacity.

When doing a family assessment, it is important to keep in mind that the assessment is of the family as a unit. The clinician must think systemically about the relationships within the family rather than focusing on the individuals making up the family unit. What matters most is how well the family functions as a system. The key is to try to understand how an individual affects the family system and how the family reacts to the individual.

A common problem in family assessment is the potential of defensiveness manifested as irritability and anger on the part of family members. This is usually a function of their sense of vulnerability and worry about being blamed for family problems. Much of this can be dealt with by a proper orientation to the assessment process which reassures the family that they are there to be heard and not to be blamed. A proper framing of the evaluation process will put the family at ease and create a collaborative environment that reduces resistance and defensiveness. If family members persist in being angry and disruptive, the family assessment should stop. The family needs to understand that without their cooperation and willingness to discuss differences and problems in a socially appropriate manner there can be no assessment.

The challenge for the clinician is to maintain control of the family meeting without being authoritarian or inhibiting a family's willingness to express their concerns. The family usually responds well if the clinician stays focused and provides a structure for the assessment. 


\section{Conclusion}

A systematic assessment of the family is central to understanding the pertinent issues in the family and its potential role in shaping a patient's presenting problem. This understanding contributes to a comprehensive biopsychosocial formulation leading to a treatment plan that is likely to address most of the variables that may need and could benefit from clinical interventions [18]. The clinician needs a consistent, broadly based and structured assessment template to be able to perform a systematic family assessment. Such a template ensures that a wide range of family dimensions are assessed and helps the clinician stay on track. There are also subjective and objective assessment instruments, in addition to the standard clinical interview, to help in the evaluation process. These instruments complement the clinical evaluation. A good family assessment can be therapeutic in and of itself even if the decision is made that no further family intervention is indicated.

\section{References}

1 Newman MC, Lawless JJ, Gelo F: Family-oriented patient care. Am Fam Physician 2007;75:9:13091310.

- Campbell TL: The effectiveness of family interventions for physical disorders. J Martial Fam Ther 2003;29:263-281.

- 3 Molloy GJ, Johnston DW, Johnston M, Morrison V, Pollard B, Bonetti D, Joice S, MacWalter R: Extending the demand-control model to informal caregiving. J Psychosom Res 2005;58:243-251.

4 Fisher L: Research on the family and chronic disease among adults: major trends and directions. Fam Syst Health 2006;24:4:373-380.

5 Fisher L, Weihs KL: Can addressing family relationships improve outcomes in chronic disease? Report of the National Working Group on Family-Based Interventions in Chronic Disease. J Fam Pract 2000; 49:561-566.

6 Ryan CE, Keitner GI: Family functioning assessment in rehabilitation and health; in Mpofu E, Oakland T (eds): Assessment in Rehabilitation and Health. Upper Saddle River, Pearson Education, 2009.

7 Hartmann M, Bazner E, Wild B, Eisler I, Herzog W: Effects of interventions involving the family in the treatment of adult patients with chronic physical diseases: a meta-analysis. Psychother Psychosom 2010;79:136-148.
8 American Academy of Family Physicians, American Academy of Pediatrics, American College of Physicians, and the American Osteoopathis Association. Joint Principles of the Patient-Centered Medical Home. March 2007. www.acponline.org/ adovacay/where_we_stand/medical_home/ approve_jp.pdf; accessed December 18, 2009.

9 Ryan CE, Epstein N, Keitner GI, Miller I, Bishop D: Evaluating and Treating Families: The McMaster Approach. New York, Routledge Taylor \& Francis Group, 2005.

10 Spanier GB: Measuring dyadic adjustment: new scales for assessing the quality of marriage and similar dyads. J Marital Fam 1976;38:15-28.

11 Beavers R, Hampson RB: Successful Families: Assessment and Intervention. New York, Norton, 1990.

12 Moos R, Moos B: Family Environment Scale Manual. Palo Alto, Consulting Psychologists Press, 1981.

13 Olson DH, Russell CS, Sprenkle DH: Circumplex Model: Systemic Assessment and Treatment of Families. New York, Haworth Press, 1989.

14 Epstein NB, Baldwin LM, Bishop DS: The McMaster Family Assessment Device. J Marital Fam Ther 1983;9:171-180.

15 Rosen KH, Mccollum EE, Middletown K, Looke L, Bird K: Interrater reliability and validity of the global assessment of relational functioning (GARF) scale in a clinical setting: a preliminary study. Am J Fam Ther 1997;25:357-360. 
16 Brown GW, Rutter M: The measurement of family activities and relationships: a methodological study. Hum Relat 1966;19:241-263.

- 17 Miller IW, Kabacoff RI, Epstein NB, Bishop DS, Keitner GI, Baldwin LM, van der Spuy HIJ: The development of clinical rating scale for the McMaster model of family functioning. Fam Process 1994;33:53-69.
18 Keitner GI, Heru AM, Glick ID: Clinical Manual of Couples and Family Therapy. Washington, American Psychiatric Publishing, 2010, pp 63-92.

\section{Gabor I. Keitner, MD}

Rhode Island Hospital, Potter Building, Room 300

593 Eddy Street

Providence, RI 02903 (USA)

Tel. +1 401444 3967, E-Mail gkeitner@lifespan.org 